Revista Perspectivas Online: Biológicas \& Saúde

Abril de 2018, Vol.8, n 26, p. 9-18

ISSN: 2236-8868 (Online)

DOI: $10.25242 / 886882620181241$

\title{
EXTRAÇÃO E CARACTERIZAÇÃo DO ÓLEO DE COCO (Cocos nucifera L.)
}

\author{
Ana Paula Santos de Pinho ${ }^{1}$ \& Aline Francisca de Souza ${ }^{1 *}$
}

PINHO, A.P.S.; \& SOUZA, A.F.; Extração e caracterização do óleo de coco (cocos nucifera 1.). Perspectivas Online: Biológicas \& Saúde. v. 8, n 26, p.9-18, 2018.

\section{RESUMO}

O presente trabalho teve como objetivo avaliar o rendimento da extração de óleo de coco de $C$. nucifera $L$. por meio de dois métodos (artesanal e com solvente orgânico) e, comparar os óleos obtidos com o óleo comercial quanto à atividade antimicrobiana e índice de acidez. Os resultados desse estudo demonstraram que as extrações analisadas foram estatisticamente equivalentes, portanto, não é possível definir o método que proporciona maior rendimento médio na obtenção de óleo de coco. Em relação à atividade antimicrobiana, os óleos obtidos por solvente orgânico, método artesanal e prensagem a frio (óleo comercial) não foram capazes de inibir o crescimento dos microrganismos $S$. aureus, E. coli $e$ C. albicans. Desta forma, os óleos analisados não apresentaram atividade antimicrobiana com relação aos microrganismos testados. $\mathrm{O}$ índice de acidez dos óleos de coco extraídos por solvente orgânico, por método artesanal e prensagem a frio demonstraram valores acima do permitido pela legislação vigente. Porém, esses valores podem estar relacionados não só com o método de extração, como também com a qualidade da matéria-prima utilizada e a conservação do produto.

Palavras-chave: Óleo vegetal, atividade antimicrobiana, índice de acidez.

\begin{abstract}
The objective of this study was to evaluate the yield of coconut oil extraction from $C$. nucifera $\mathrm{L}$. using two methods (artisanal and with organic solvent) and to compare the oils obtained with the commercial oil for the antimicrobial activity and the index of acidity. The results of this study demonstrated that the analyzed extractions were statistically equivalent, therefore, it is not possible to affirm the method with higher average yield of coconut oil. Regarding the antimicrobial activity, the oils obtained by organic solvent, artisanal

not able to inhibit the growth of $S$. aureus, E. coli and $C$. albicans microorganisms. Thus, the oils analyzed did not present antimicrobial activity in relation to the tested microorganisms. The acidity index of coconut oils extracted by organic solvent, using a hand-held method and cold pressing, showed values higher than allowed by current legislation. However, these values may be related not only to the extraction method, but also to the quality of the raw material, used and the conservation of the product.
\end{abstract} method and cold pressing (commercial oil) were

Keywords: Vegetable oil, antimicrobial activity, acidity index.

${ }^{1}$ Centro Universitário Teresa D’Ávila - Av. Dr. Peixoto de Castro, 539 - Cruz, Lorena - CEP: 12606-580 - SP.

(*)e-mail: alinefsmga@gmail.com

Data de chegada: 23/07/2017 Aceito para publicação: 13/04/2018 


\section{INTRODUÇÃO}

O coqueiro (Cocos nucifera, L.) é cultivado em mais de 86 países situados nos trópicos, tanto para consumo do fruto, como para fins industriais (FIGUEIRA, 2012). No Brasil, o coqueiro foi introduzido em 1553, no Estado da Bahia, a partir de material trazido de Cabo Verde (ARAGÃO et al., 2010). Essa planta disseminou-se para o litoral nordestino, onde encontrou clima favorável para o seu cultivo, posteriormente adaptou-se em outras regiões do Brasil (MARTINS; JESUS-JUNIOR, 2011).

Em 2001, a produção brasileira de coco chegou a 1,3 bilhões de frutos e nos últimos anos registrouse um acréscimo dessa produção. Em 2016, esperava-se o rendimento de 7.979 frutos por hectare, em uma área de 231.087 hectares plantados, o que corresponderia a 1.8 bilhões de frutos (IBGE, 2016).

O coco produzido pode ser utilizado pelas indústrias a partir do processamento do endosperma sólido submetido à secagem (copra) ou fresco. O coco fresco é o mais utilizado no Brasil na indústria alimentícia para fabricação de leite de coco, coco ralado, doces, bolos, bombons, chocolates, entre outros. A fibra de coco, derivada do mesocarpo do fruto, pode ser utilizada na confecção de tapetes, enchimentos de bancos de automóveis e pó para substrato agrícola (FONTENELE, 2005).

O óleo de coco é derivado da copra, e contém grande quantidade de lipídeos de baixo peso molecular como ácido láurico (MARINA et al., 2009). Segundo Araújo (2008), no óleo de coco pode-se encontrar ácido caprílico, cáprico, mirístico, palmítico, esteárico, oléico, linoleico e ácido láurico, sendo esse último encontrado em maior proporção. Outros componentes encontrados em menores concentrações incluem monoglicerídeos, diglicerídeos, fosfatídeos, ceras, pigmentos (carotenóides e clorofila) e esteróis (matéria insaponificável) como os tocoferóis, que inibem a oxidação das cadeias de ácidos graxos insaturados do óleo de coco (MORETTO; FETT 1998).

Estudos revelam que os polifenóis presentes no extrato da fibra da casca de coco (C. nucifera) apresentam atividade antiparasitária contra Leishmania amazonensis (MENDONÇA-FILHO et al., 2004), atividades antibacteriana (Staphylococcus aureus), antifúngica (Candida albicans, Fonsecaea pedrosoi e Cryptococcus neoformans) e antiviral contra vírus herpes simplex tipo 1 (ESQUENAZI et al., 2002). O consumo regular de óleo de coco pode reduzir a concentração de colesterol total, triglicerídeos, fosfolipídeos, lipoproteínas de baixa densidade (LDL- Low density lipoprotein), bem como aumentar o nível de lipoproteínas de alta densidade (HDL - High density lipoprotein) no soro sanguíneo, reduzindo os riscos de doenças cardiovasculares (NEVIN; RAJAMOHAN, 2004).

Experimentos realizados por Figueira (2012) com extratos do pecíolo e folhas de $C$. nucifera $L$. demonstraram a inibição do crescimento bacteriano de Staphylococcus aureus e P. aeruginosa, porém não foi observada a inibição do crescimento de E. coli e C. albicans. Almeida (2012), demonstrou que a utilização do óleo de coco extraído de forma artesanal e industrializado no tratamento de feridas in vivo pode inibir o crescimento de $S$. aureus, porém apenas o óleo de coco extraído de forma artesal foi capaz de inibir o | crescimento de S. epidermidis e Enterobacter cloacae.

Existem vários métodos para a extração de óleos, no entanto, os principais são a extração artesanal, mecânica e por solvente (GONZALEZ et al., 2008). No método de extração artesanal a polpa do fruto é submetida ao cozimento intensivo em um recipiente contendo água. Após a fervura, o óleo sobrenadante é separado da parte aquosa. Em seguida, ocorre a secagem do óleo em fogo baixo, com a utilização de um recipiente metálico sobre uma chapa quente até que o óleo perca a opacidade devido à umidade, após esse procedimento, o óleo é filtrado em papel de filtro (FACIOLLI \& GONÇALVES, 1996). De acordo com Carvalho (2011), o método artesanal apresenta alguns inconvenientes, como baixo rendimento, produto com aspecto opaco e alta umidade. Para evitar o processo de oxidação em poucos dias, o óleo de coco artesanal deve que ser centrifugado, o que proporciona melhor separação da fase oleosa e resíduos da extração.

Persp. online: biol. \& saúde, Campos dos Goytacazes, 26 (6), 9-18, 2018

seer.perspectivasonline.com.br 
A extração mecânica pode ser realizada pela técnica de prensagem, sem a utilização de solventes, desta forma, o produto obtido mantém suas propriedades naturais. Porém, devido a sua menor eficiência na retirada de óleo da fruta ou semente, essa extração pode ser combinada à extração por solvente para aumentar o rendimento. Quando realiza a prensagem com alta pressão, o conteúdo de óleo residual na torta é reduzido em 5\%, dispensando o subsequente uso do solvente (MORETTO; FETT, 1998). A extração por prensagem apresenta menor custo de extração, devido ao fato de não utilizar energia elétrica e solventes, como também não gera aquecimento do óleo e da torta, sendo necessário apenas uma prensa e um cilindro extrator (CARVALHO, 2011).

A extração por solvente consiste em colocar um solvente orgânico em contato direto com a matriz vegetal. Após um intervalo de tempo suficiente para que ocorra a transferência dos constituintes solúveis presentes na planta, efetua-se a separação das fases sólida e líquida. O óleo é obtido pela evaporação do solvente presente na fase líquida (STEFFANI, 2003). Essa metodologia é indicada para óleos que são muito instáveis, não suportando aumentos de temperatura. Neste processo os solventes orgânicos mais utilizados para extração são o hexano, benzeno, metanol, etanol, propanol, acetona, pentano e diversos solventes clorados (FILIPPIS, 2001).

Um dos efeitos negativos da utilização de solventes orgânicos é a dificuldade na remoção de todo o solvente residual e a extração de compostos não voláteis. Para remoção destes compostos é necessária muita energia e alto investimento em equipamentos, além disso, os solventes podem provocar alterações químicas nas moléculas e efeitos tóxicos nos consumidores (SARTOR, 2009). Pesquisas realizadas por Carvalho (2011), demonstraram que a extração de óleo de Mauritia flexuosa L.f. com o solvente Hexano P.A. apresentou maior rendimento $(23,55 \%)$, quando comparado com a extração por prensagem hidráulica $(21,50 \%)$ e por método artesanal $(4,01 \%)$. De acordo com o mesmo autor, a extração por solvente proporciona maior rendimento, porém produz resíduos químicos, utiliza energia e gera aquecimento tanto no óleo quanto na torta.

Ao considerar os diversos benefícios proporcionados pelo óleo de coco e que o método de extração pode influenciar no rendimento, na composição química e nas propriedades bioativas do óleo, o presente trabalho teve como objetivo avaliar o rendimento da extração de óleo de coco de $C$. nucifera, $L$. por método artesanal e por método com solvente orgânico e comparar com o óleo comercial extraído por prensagem a frio, com relação à atividade antimicrobiana e índice de acidez.

\section{MATERIAL E MÉTODOS}

\section{2 .1 Obtenção da matéria-prima}

O fruto $C$. nucifera L. foi obtido em unidades secas, no comércio do município de Cruzeiro-SP. O coco foi quebrado, ralado, acondicionado em embalagens plásticas estéreis, e armazenados a $4^{\circ} \mathrm{C}$ no Laboratório de Microbiologia do Centro Universitário Teresa D’Ávila para posterior extração do óleo.

\section{2 .2 Extração de óleo de coco por solvente orgânico}

A extração do óleo de coco foi realizada com solvente orgânico de acordo com o método modificado de Fuller (2008) e Steffani (2003). Para tanto, foram adicionados $90 \mathrm{~mL}$ de hexano a $30 \mathrm{~g}$ de coco seco ralado, ambos permaneceram em repouso por 72 horas. Após esse período procedeu-se à separação do solvente através de em um aparelho rota vapor à temperatura de $60^{\circ} \mathrm{C}$. O óleo obtido foi armazenado a $4^{\circ} \mathrm{C}$ para posteriores análises. 


\section{2 .3 Extração de óleo de coco por método artesanal}

A extração de óleo de coco pelo método artesanal foi realizada de acordo com metodologia modificada de Santos et al. (2013). Foram adicionados $210 \mathrm{~g}$ de polpa de coco $C$. nucifera $\mathrm{L}$. cortada em cubos a $210 \mathrm{~mL}$ de água destilada e batidos em um liquidificador. Após esse processo, a amostra foi filtrada e espremida em gaze, a fim de extrair o máximo de solução líquida possível. A fração aquosa permaneceu em repouso por 24 horas para decantação. Após o período de repouso, o sobrenadante da amostra foi separado, acondicionado em um frasco metálico e aquecido em bico de Bunsen para separação do óleo de coco. O óleo obtido foi armazenado em um recipiente plástico a $4^{\circ} \mathrm{C}$ para posterior análise.

\section{2 .4 Obtenção do óleo comercial extraído por prensagem a frio}

O óleo de coco industrial foi obtido no comércio local, e a marca escolhida para realização dos experimentos foi COPRA $®$. De acordo com dados fornecidos pela empresa, a extração do óleo foi realizada pelo método de prensagem a frio, e a acidez máxima do produto indicada no rótulo é igual a $0,3 \%$.

\section{2 .5 Rendimento do extrato de óleo de coco}

O rendimento total do óleo de coco extraído de Cocos nucifera L. foi calculado a partir da razão entre o total de extrato obtido (óleo) e a massa inicial do coco utilizado.

\section{2 .6 Análise de acidez}

A análise do índice de acidez do óleo de coco foi executada de acordo com a metodologia modificada de Silva et al. (2010). Para tanto, 2,5g de hidróxido de sódio (NaOH) foram dissolvidos em 250 $\mathrm{mL}$ de água, para a obtenção da concentração $0,25 \mathrm{~mol} / \mathrm{L}$. Em outro frasco, foram pesados $1,75 \mathrm{~g}$ de óleo de coco em $75 \mathrm{~mL}$ de etanol. A amostra foi agitada para dissolução e em seguida foram adicionados $2 \mathrm{~mL}$ de fenolftaleína $1 \%$ como indicador de $\mathrm{pH}$. A titulação foi realizada com o gotejamento da solução de $\mathrm{NaOH}$ $0,25 \mathrm{~mol} / \mathrm{L}$ à amostra contendo óleo de coco, etanol e fenolftaleína até atingir uma coloração levemente rósea, por no mínimo, 15 segundos.

\section{2 .7 Avaliação da atividade antimicrobiana}

Foram utilizados micro-organismos padrões cedidos pelo laboratório de Microbiologia do Centro Universitário Teresa D’Ávila, Lorena-SP. As cepas de Escherichia coli (ATCC 25922), Staphylococcus aureus (ATCC 25923) e Candida albicans (ATCC 10231) foram ativadas em caldo nutriente e repicadas por dois dias consecutivos, incubadas a $37^{\circ} \mathrm{C}$ para posterior análise da eficiência da atividade antimicrobiana e antifúngica de óleo de coco extraído pela técnica artesanal, com solvente orgânico e prensado a frio (óleo comercial).

A avaliação da atividade antimicrobiana, foi realizada pelo método adaptado de Silva (2011), na qual suspensões dos micro-organismos avaliados foram semeadas na superfície de placas de Petri contendo o meio de cultura ágar nutriente. Posteriormente foram adicionados na placa de Petri, 5 discos de papel filtro previamente embebidos em óleo de coco. As placas foram incubadas a $37^{\circ} \mathrm{C}$ por 48 horas. Os testes foram realizados em triplicata.

Após 48 horas de incubação, as placas foram examinadas para verificar a uniformidade do crescimento microbiano, bem como a presença de contaminantes e a definição do diâmetro do halo de inibição, conforme metodologia prescrita por Moreira et al. (2009). 


\section{2 .8 Análise estatística}

Os resultados dos experimentos foram analisados estatisticamente pelo Teste t de Student, utilizando o programa Statistica 5, considerando o nível de 10\% de significância.

\section{RESULTADOS}

\section{3 .1 Rendimento do extrato de óleo de coco}

Os dados e resultados do rendimento obtidos a partir da extração de óleo de coco (Cocos nucifera, L.) por solvente orgânico utilizando hexano e por método artesanal são mostrados na Tabela 1. Pode-se observar que os intervalos de confiança para a extração com solvente foram de 0,0899 a 0,1288 mL/g, enquanto que para a extração artesanal foram de 0,0734 a $0,2306 \mathrm{~mL} / \mathrm{g}$.

De acordo com a análise estatística, os resultados do rendimento da extração de óleo de coco, obtidos a partir do método com solvente orgânico e do método artesanal, apresentaram resultados não significativos (valor de $p$ igual a 0,2503), sendo considerados estatisticamente equivalentes, ao nível de $10 \%$ de significância.

Tabela 1. Rendimento da extração de óleo de coco (Cocos nucifera, L.) por solvente orgânico e por método artesanal.

\begin{tabular}{lcccccc}
\hline $\begin{array}{c}\text { Tipo de } \\
\text { Extração }\end{array}$ & $\begin{array}{c}\text { Polpa } \\
\text { Ralada } \\
(\mathbf{g})\end{array}$ & $\begin{array}{c}\text { Rendimento } \\
\text { em óleo }(\mathbf{m L})\end{array}$ & $\begin{array}{c}\text { Rendimento } \\
(\mathbf{m L} / \mathbf{g})\end{array}$ & $\begin{array}{c}\text { Rendimento } \\
\text { Médio } \\
(\mathbf{m L} / \mathbf{g})\end{array}$ & $\begin{array}{c}\text { Desvio } \\
\text { Padrão } \\
(\mathbf{m L} / \mathbf{g})\end{array}$ & $\begin{array}{c}\text { Intervalo de } \\
\text { confiança }(\mathbf{m L} / \mathbf{g})\end{array}$ \\
\hline \multirow{3}{*}{ Solvente } & 30 & 2,9 & 0,096 & & & \\
& 30 & 3,5 & 0,116 & 0,109 & 0,0115 & $0,0899-1,288$ \\
& 30 & 3,5 & 0,116 & & & \\
Artesanal & 210 & 40 & 0,190 & & & \\
& 210 & 35 & 0,166 & 0,152 & 0,0466 & $0,0734-0,2306$ \\
& 210 & 21 & 0,100 & & & \\
\hline
\end{tabular}

\section{3 .2 Avaliação da atividade antimicrobiana}

Após a extração e avaliação do rendimento, os óleos de coco obtidos de forma artesanal e com solvente orgânico foram submetidos à avaliação da atividade antimicrobiana com relação à inibição do crescimento das cepas E. coli, S. aureus e C. albicans. O óleo de coco comercial da marca COPRA®, extraído por prensagem a frio, também foi analisado pela mesma metodologia. Os resultados da avaliação da atividade antimicrobiana demonstraram que o óleo de coco extraído de forma artesanal, com solvente orgânico e o óleo comercial extraído por prensagem a frio não foram capazes de inibir o crescimento microbiano, indicando que independente do método de extração e das características químicas, os óleos de coco avaliados não apresentaram atividade antimicrobiana.

\section{3 .3 Determinação do índice de acidez}

Os óleos de coco extraídos com solvente orgânico, pelo método artesanal e o óleo comercial foram submetidos à avaliação de índice de acidez. Os resultados expressos em \% de ácido oleico e mg de NaOH/g são mostrados na (Tabela 2). 
Tabela 3. Índice de acidez do óleo de coco extraído por método artesanal, solvente orgânico e por prensagem a frio (comercial) expresso em \% ácido oleico e em $\mathrm{mgNaOH} / \mathrm{g}$

\begin{tabular}{ccc}
\hline Tipo de extração & Índice de acidez em \% de ácido oleico & Índice de acidez $(\mathbf{m g N a O H / g )}$ \\
\hline Artesanal & 2,09 & 4,16 \\
Solvente orgânico & 2,79 & 5,56 \\
Prensagem à frio & 2,09 & 4,16 \\
\hline
\end{tabular}

Fonte: Arquivo do autor.

De acordo com os parâmetros estipulados pela legislação (ANVISA, 2005) e as Normas do Codex Alimentarius - FAO/OMS, os valores máximos de acidez permitidos são de 2,0\% ácido oleico e 4,0 de $\mathrm{mgNaOH} / \mathrm{g}$. Os resultados da Tabela 2 demonstraram que a acidez dos óleos de coco extraídos por solvente orgânico, pelo método artesanal e por prensagem a frio (comercial) estão acima do recomendado. A extração do óleo por solvente orgânico proporcionou maior índice de acidez $(2,79 \%$ de ácido oleico ou 5,56 $\mathrm{mg} / \mathrm{NaOH}$ ), quando comparado aos métodos de extração artesanal ou por prensagem a frio, que apresentaram índice de acidez de 2,09\% de ácido oleico ou 4,16 mgNaOH/g. De acordo com Machado et al. (2006), o índice de acidez indica o estado de conservação do óleo. O processo de refino pode reduzir a acidez, enquanto que o aquecimento e a luz podem acelerar a decomposição do óleo, causando a rancidez, que quase sempre é acompanhada pela formação de ácidos graxos livres.

\section{DISCUSSÃO}

O processo de extração de óleos vegetais vem sofrendo mudanças significativas a fim de elevar a eficiência, diminuir o grande consumo de energia e o impacto ambiental devido aos resíduos gerados no processo de produção. Estas mudanças têm sido realizadas em todas as etapas do processo, que incluem desde a preparação das sementes (matéria-prima) até o processo final da extração e obtenção do óleo (GONZALEZ, 2008). Um parâmetro importante a ser considerado na extração do produto é a relação rendimento e custo benefício, uma vez que o maior rendimento e menor custo proporcionará maior vantagem econômica ao produto.

Os resultados dos métodos de extração analisados demonstraram que as amostras de óleo de coco extraídas pelo método artesanal apresentaram maiores rendimentos (média $0,152 \mathrm{~mL} / \mathrm{g}$ ), quando comparado com a média amostral extraída por solvente orgânico $(0,109 \mathrm{ml} / \mathrm{g})$. Porém, a análise estatística não demostrou diferença significativa entre os valores, com nível de confiança de $90 \%$ pelo Teste t de Student, com valor de $p$ igual a 0,2503 , sendo considerados, valores equivalentes, portanto, não é possível definir o método que proporciona maior rendimento médio de extração. Esses dados se contrapõem aos resultados obtidos por Carvalho (2011), na extração do óleo de Mauritia flexuosa L.f. (ARECACEAE - buriti), onde foram relatados rendimentos de $23,55 \%$ na extração realizada com hexano como solvente orgânico e 4,01 \% na extração realizada a partir do método artesanal. Os dados obtidos evidenciaram a necessidade da realização de novos estudos com maior número de amostras, sendo esta uma das limitações da pesquisa.

De acordo com Carvalho (2011), a extração com solvente é uma metodologia que gera resíduos químicos e apresenta grande gasto de energia. Por outro lado, o método artesanal, apesar de oneroso, não necessita de equipamentos específicos, não gera resíduos químicos ou gasto de energia. Os resultados obtidos no presente trabalho demonstraram que o processo de extração artesanal além de ser uma metodologia mais vantajosa ambientalmente, apresentou maior rendimento na extração, quando comparada com a extração por solvente, porém é necessário realizar novos estudos para avaliar o custo econômico dessa metodologia de extração.

As propriedades antimicrobianas de metabólitos produzidos por plantas a partir do seu metabolismo secundário têm sido reconhecidas empiricamente durante séculos, mas a busca e verificação de sua eficácia

Persp. online: biol. \& saúde, Campos dos Goytacazes, 26 (6), 9-18, 2018

seer.perspectivasonline.com.br 
científica são recentes. Por outro lado, os microrganismos que causam prejuízos à saúde humana estão se mostrando cada vez mais resistentes a maioria dos antimicrobianos conhecidos, o que incentiva ainda mais a procura por antibióticos de ocorrência natural (DUARTE, 2006). Assim, a avaliação da atividade antimicrobiana do óleo de coco representa uma contribuição para a pesquisa sobre novos antimicrobianos, principalmente pelo método artesanal, que é de fácil aplicação e desenvolvimento e não gera resíduos que podem provocar impactos ambientais.

Diversos autores relataram que as raízes, folhas, pecíolo e o óleo de $C$ nucifera $\mathrm{L}$. apresentam atividade antimicrobiana (SIVAKUMA et al., 2012; FIGUEIRA, 2012; ALMEIDA, 2012). Experimentos realizados por Sivakumar et al., (2011) demonstraram que o extrato da raiz de $C$. nucifera L. inibiu o crescimento de $S$. aureus e $P$. aeruginosa. Figueira (2012), demonstraram que extratos brutos de folha, caule e raiz de $C$. nucifera $\mathrm{L}$. apresentam atividade antimicrobiana com relação a $P$. aeruginosa e $S$. aureus. Segundo experimentos realizados por Almeida (2012), o óleo de coco extraído de forma artesanal e industrializado apresentou atividade antimicrobiana in vivo com relação ao crescimento de $S$. aureus, porém apenas o óleo de coco extraído de forma artesal foi capaz de inibir o crescimento de S. epidermidis e Enterobacter cloacae. Os mesmos resultados foram confirmados in vitro pelo autor, demonstrando que extratos oleosos artesanais e o óleo comercial extraído por prensagem a frio apresentaram efeito antimicrobiano contra $S$. Aureus, S. epidermidis e E. cloacae. Apesar de vários autores relatarem atividade antimicrobiana em extratos obtidos $C$ nucifera $\mathrm{L}$., os experimentos realizados no presente trabalho com óleo de coco extraído pelo método artesanal por solvente orgânico e por prensagem a frio (óleo comercial), não demonstraram inibição do crescimento de Escherichia coli, Staphylococcus aureus e Candida albicans, não sendo efetivo contra tais microrganismos.

Segundo Lutz (2004), a determinação da acidez fornece um dado importante na avaliação do estado de conservação do óleo. De acordo com ANVISA (2005), os padrões estipulados pela Resolução RDC n $^{\circ} 270$, o índice de acidez (I.Ac.) de óleos não deve exceder o limite máximo de 4,0 mgNaOH/g ou no máximo 2,0\% de ácido oleico. A resolução destaca ainda, que a identidade de óleos vegetais, incluindo azeites de oliva, e de gorduras vegetais deve atender também aos requisitos de composição determinados em Normas do Codex Alimentarius - FAO/OMS, que estabelece parâmetros de I.Ac. de 4,0 $\mathrm{mgNaOH} / \mathrm{g}$ para óleos prensados a frio e não refinados. De acordo com Pereira (2007), vários fatores podem influenciar a acidez de um óleo vegetal, sendo o principal o tratamento dado ainda às sementes durante a colheita e armazenamento. Portanto, a acidez está diretamente relacionada com a qualidade da matéria-prima, com o processamento e, principalmente, com as condições de conservação dos óleos vegetais. Desta forma, a acidez do óleo de coco detectada no presente trabalho, com valores acima dos parâmetros exigidos pela legislação vigente, pode estar relacionada não só com o método de extração, como também com a qualidade da matéria-prima utilizada e a conservação do produto. A alteração na acidez também ter influenciado a atividade antimicrobiana com relação aos micro-organismos testados, porém mais estudos precisam ser realizados.

\section{CONCLUSÕES}

Os óleos analisados não podem ser considerados antimicrobianos contra as cepas avaliadas uma vez que os óleos obtidos pelos diferentes métodos não foram capazes de inibir o crescimento dos microrganismos S. aureus, E. coli e C. albicans.

\section{REFERÊNCIAS}

ALMEIDA L. C. T.; TENÓRIO L. M. M. C.; VERISSÍMO R. C. S. S.; LÚCIO I. M. L.; BASTOS M. L. A, 2012. Potencial Antimicrobiano do Óleo de Coco no Tratamento de Feridas. Revista da Rede de Enfermagem do Nordeste. 13(4):880-7.

AGÊNCIA DE VIGILÂNCIA SANITÁRIA-ANVISA. Regulamento Técnico Para Óleos Vegetais, Gorduras Vegetais e Creme Vegetal. Resolução RDC n. 270, de 22 de setembro de 2005. Diário Oficial da República Federativa do Brasil, Brasília, DF, 23 de set. 2005.

Persp. online: biol. \& saúde, Campos dos Goytacazes, 26 (6), 9-18, 2018

seer.perspectivasonline.com.br 
ARAGÃO, W. M.; RIBEIRO, MELO, M. F., 2009.Cultivares de coqueiro para a produção de coco seco: coqueiro Gigante vs híbridos. In: CINTRA, F. L. D.; FONTES, H. R.; PASSOS, E. E. M.; FERREIRA, J. M. S. (Ed.). Fundamentos tecnológicos para a revitalização das áreas cultivadas com coqueiro gigante no nordeste do Brasil. Aracaju: Embrapa Tabuleiros Costeiros,232 p. p. 37- 60.

ARAÙJO, J. S, 2008. Produção de Biodiesel a partir de óleo de coco (Cocos nucifera L). UFRN. Natal.

BARUFFALDI, R.; OLIVEIRA, M. N, 1998. Fundamentos de tecnologia de alimentos. v.3, São Paulo: Atheneu.

CARVALHO, C. O, 2011. Comparação entre métodos de extração do óleo de Mauritia flexuosa 1.f. (ARECACEAE - buriti) para o uso sustentável na reserva de desenvolvimento tupé: rendimento e atividade antimicrobiana. Dissertação (Mestrado em Biotecnologia e Recursos Naturais) - Universidade do Estado do Amazonas, Manaus.

DAUBER, A. R, 2015. Óleo de Coco: Uma Revisão Sistemática. Universidade Federal do Rio Grande do Sul. Faculdade de Medicina. Porto Alegre.

Duarte, M. C. T, 2006. Atividade Antimicrobiana de Plantas Medicinais e Aromáticas Utilizadas no Brasil. MultiCiência: Construindo a história dos produtos naturais; 7:1-16.

ESQUENAZI, D.; WIGG, M. D.; MIRANDA, M. M. F. S.; RODRIGUES, H. M.; TOSTES, J. B. F.; ROZENTAL, S.; SILVA, A. J. R.; ALVIANO, C. S, 2002. Antimicrobial and antiviral activities of polyphenolics from Cocos nucifera Linn. (Palmae) husk fiber extract, Research in Microbiology, v. 153, p.647-652.

FACIOLLI, N. L.; GONÇALVES, L.A.G, 1996. Modificação por via enzimática da composição triglicerídica do óleo de pequi (Caryocar brasiliensi Camb.). Departamento de Tecnologia de Alimentos Faculdade de Engenharia de Alimentos - UNICAMP - 13.083-970 - Campinas -SP.

FERREIRA, M. S.; WARWICK, D. R. N.; SIQUEIRA, L. A, 1997. A cultura do coqueiro no Brasil. Brasília: Embrapa/SPI; Aracaju: Embrapa/CPATC.

FIGUEIRA, C. N. T, 2012. Avaliação da atividade antimicrobiana, citotóxica e capacidade sequestradora de radicais livres de extratos brutos do Cocos nucifera L. Dissertação Mestrado. Maceió-AL.

FILIPPIS, F. de M, 2001. Extração com CO2 supercrítico de óleos essencial de Hon- sho e Ho-shoexperimentos e modelagem. Dissertação (Mestrado em Engenharia Química) - Departamento de Engenharia Química, Universidade Federal do Rio Grande do Sul.

FÜLLER, T. N, 2008. Caracterização fenotípica, fitoquímica e molecular de populações de Elionurus SP. Humb. \& Bompl ex Willd (capim-limão). Dissertação (Mestrado em Fitotecnia). Falculdade de Agronomia Universidade Federal do Rio Grande do Sul. Porto Alegre.

FONTENELE, R. E. S. CULTURA DO COCO NO BRASIL, 2005. CARACTERIZAÇÃO DO MERCADO ATUAL E PERSPECTIVAS FUTURAS, XLIII CONGRESSO DA SOBER, Sociedade Brasileira de Economia e Sociologia Rural.

GONZALEZ, W.A, 2008. Biodiesel e óleo vegetal in natura. Brasília: Ministério de Minas e Energia, Gurupá - Pará. Relatório de Visita Técnica.

Persp. online: biol. \& saúde, Campos dos Goytacazes, 26 (6), 9-18, 2018

seer.perspectivasonline.com.br 
HANN V.B.; MARTINS M. S.; DIAS R. L, 1981. Termogênicos: Uma revisão sistemática sobre o uso de óleo de coco, óleo de cártamo e CLA. Revista Brasileira de Nutrição Esportiva, São Paulo. v. 8. n. 43. p.1019. Jan/Fev. 2014. ISSN 9927.

IBGE. Levantamento sistemático da produção agrícola. Disponível em: ftp://ftp.ibge.gov.br/Producao_Agricola/Levantamento_Sistematico_da_Producao_Agricola_[mensal]/Fascic ulo/lspa_201609.pdf. Acesso em 21 de set. de 2016.

INSTITUTO ADOLFO LUTZ, Métodos Físico-Químicos para Análise de Alimentos. $4^{\mathrm{a}}$ Edição. $1^{\mathrm{a}}$ Edição Digital. São Paulo, 2008.

MACHADO, G. C.; CHAVES, J. B. P; ANTONIASSI, R. 2006. Composição em ácidos graxos e caracterização física e química de óleos hidrogenados de coco babaçu. Revista Ceres, v. 53, n. 308, p. 463470.

MARINA, A. M.; CHEMAN, Y. B.; NAZIMAH, S. A.H., AMIN, I, 2009. Chemical Properties of Virgin Coconut Oil, Journal of the American Oil Chemists' Society, v. 86, p. 301-307.

MARTINS, C. R. M.; JESUS JUNIOR, L. A, 2011. Evolução da produção de coco no Brasil e o comércio internacional - Panorama 2010, EMBRAPA.

MENDONÇA-FILHO, R, R.; RODRIGUES, I. A.; ALVIANO, D. S.; SANTOS, A. L. S.; SOARES, R. M. A.; ALVIANO, C. S.; LOPES, A. H. C. S.; ROSA, M. S, 2004. Leishmanicidal activity of polyphenolic-rich extract from husk fiber of Cocos nucifera Linn. (Palmae). Research in Microbiology, v. 144, p. 136-143.

MOREIRA, A. C. A. et al., Avaliação in vitro da atividade antimicrobiana de bucais R. Ci. méd. biol., Salvador, v.8, p.153-161, 2009.

MORETTO, E.; FETT, R, 1998. Definição de óleos e Gorduras tecnologia de óleos e gorduras vegetais na indústria de alimentos. São Paulo.

NEVIN, K. G.; RAJAMOHAN, T, 2004. Beneficial effects of virgin coconut oil on lipid parameters and in vitro LDL oxidation. Clinical Biochemistry, v. 37, p. 830-835.

PEREIRA, A. F. C. 2007. Determinação simultânea de acidez, índice de refração e viscosidade em óleos vegetais usando espectrometria NIR, calibração multivariada e seleção de variáveis. Dissertação de mestrado. Universidade Federal da Paraíba. Centro de ciências exatas e da natureza. Departamento de Química. Pós-Graduação em Química.

SANTOS, J. R. M.; MARTINS, J. S.; FREIRE, M. S.; NETO, N. S.; SILVA, T. N, 2013. Caracterização físico-química do óleo de coco obtido artesanalmente. Universidade Federal de Campina.

SARTOR, R. B.; 2009. Modelagem, Simulação e Otimização de uma Unidade Industrial de Extração de Óleos Essenciais por Arraste a Vapor. Dissertação (Mestrado em Pesquisa e Desenvolvimento de Processos). Escola de Engenharia, Universidade Federal do Rio Grande do Sul. Porto Alegre.

SERAFINI, L.A.; SANTOS, A.C.A.; TOUGUINHA, L.A.; AGOSTINI, G.; DALFOVO, V, 2002. Extrações e aplicações de óleos essenciais de plantas aromáticas e medicinais. Caxias do Sul: EDUCS.

SILVA, M. G. F, 2011. Atividade antioxidante e antimicrobiana in vitro de óleos essenciais e extratos hidroalcóolicos de manjerona (Origanum majorana L.) e manjericão (Ocimum basilicum L.). Trabalho de Conclusão de Curso - Curso Superior de Química - Bacharelado em Química Industrial/Licenciatura em Química, Universidade Tecnológica Federal do Paraná. Pato Branco. 
SILVA, E. P.; SILVA, H. M. G.; ALMEIDA, R. S.; MONTEIRO, E. A.; ROCHA, T. M, 2010. Determinação do índice de acidez em óleo de milho para produção de biodiesel. Instituto Federal de Educação, Ciências e Tecnologia do Maranhão.

SIVAKUMAR, M. K.; MOIDEEN, M. M.; VARGHESE, R.; SHEIK, B.; DHANAPAL, C. K, 2011. Preliminary phytochemical screening and anti-bacterial activity of Cocos nucifera Linn root. Karpagam University, Coimbatore, Tamil Nadu, India.

STEFFANI, E, 2003. Modelagem matemática do processo de extração supercrítica de óleo essencial de HoSho (Cinnamomum camphora Nees, Eberm var. linaloolifera Fujita) Utilizando $\mathrm{CO}_{2}$. Tese (Doutorado) Universidade Federal de Santa Catarina. 